\title{
Characterization of Ion Acceleration Processes in a Surface ECR Plasma Source
}

\author{
Bradley S. Sommers ${ }^{1}$, and John E. Foster ${ }^{2}$ \\ University of Michigan, Ann Arbor, MI, 48104
}

\begin{abstract}
A microwave ECR ion source is developed using distributed ECR antenna excitation over the cusp of a permanent multipole magnetic configuration. The ion source employs a combination of natural nozzle acceleration effects occurring within the cusp and the application of an external DC bias to extract both electron and ion current from the device. The ECR discharge is shown to be stable over the pressure range 0.5-100 mTorr and at low pressure (1.4 mTorr), beam energies up to $80 \mathrm{eV}$ are observed. In this paper, we present both current extraction and ion beam energy measurements taken over the pressure range 1-20 mTorr and power levels between 20 and $40 \mathrm{~W}$. In addition, beam energy and beam energy spread are documented and correlated to discharge voltage.
\end{abstract}

\section{Nomenclature}

$e \quad=$ electronic charge

$M \quad=$ Argon ion mass

$A=$ Area of RPA collector

$\tau=$ Transmission coefficient for RPA

$I_{c} \quad=$ RPA collector current

$\varphi=$ RPA discriminator voltage

$\mathrm{f}(\mathrm{e} \varphi) \quad=$ ion energy distribution

$\mathrm{C}=$ Conductance of exposed slot aperture

$\mathrm{P}_{\text {out }}=$ Background vacuum chamber pressure

$\mathrm{P}_{\text {in }} \quad=$ Internal device pressure

$\mathrm{Q}=$ Throughput

$\mathrm{w}_{\mathrm{ce}} \quad=$ Electron cyclotron frequency

$\mathrm{w}_{\mathrm{pe}} \quad=$ Electron plasma frequency

$v_{\mathrm{e}} \quad=$ Electron collision frequency

$(<\sigma v>)=$ Ionization product

$\mathrm{n}_{0} \quad=$ neutral density

\section{Introduction}

Magnetic multipole configurations have been used to enhance plasma confinement in a variety of plasma applications, particularly in plasma processing and electric propulsion ${ }^{1}$. Traditionally, these multipole sources are composed of lines or rings of magnets that form a series of alternating polarity magnetic cusps. The high magnetic field strength in these cusps increases the containment lifetime of primary plasma electrons, allowing them a

\footnotetext{
1 Research Assistant, Dept. Nuclear Engineering, 2355 Bonisteel Blvd. Ann Arbor, MI, 48104.

2 Associate Professor, Dept. Nuclear Engineering, 2355

Bonisteel Blvd. Ann Arbor, MI, 48104
}

higher probability of causing ionization. This effect has been shown to improve both utilization efficiency and plasma spatial uniformity within the device ${ }^{2}$. Plasma sources with magnetic cusps typically utilize permanent magnets. The magnetic field structure of these permanent magnets, shown in fig. 1a, has the appearance of a nozzle or funnel, with field lines spreading out and decreasing in magnitude with distance above the pole. This field gradient provides a mirror force that limits current collection on the magnet surface to only the small fraction of electrons that fall into its loss cone. All other electrons are reflected away, forced to bounce back and forth between adjacent cusps, following field lines similar to those shown in fig. $1 \mathrm{~b}$. (a)

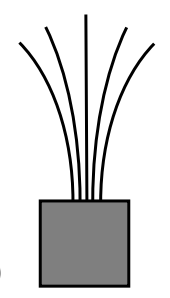

(b)

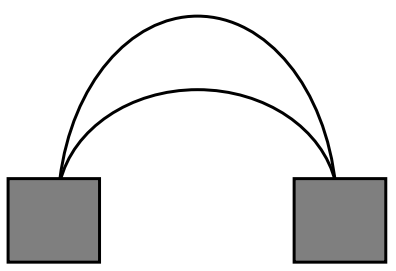

Figure 1. Magnetic field geometry over a cusp. a.) In a single cusp, the field diverges out with decreasing field strength. b.) Field lines joining two magnetic cusps of opposite polarity.

If a plasma were produced directly inside the cusp, it would tend to expand away from the surface of the magnet, driven by a combination of the mirror force and a concentration gradient. This plasma would therefore escape the confinement of the field, flowing out along the field lines, eventually detaching from the field lines as the Larmor radius 
exceeds the characteristic length scale of the plasma source. The energy of these exiting ions or electrons could be controlled and increased up to significant beam energies ( $\sim 100$ s of volts) if a sufficiently high potential difference could be established across the source plasma. Since the strong axial field gradients prevent the electrons from reaching the anode (the cusp surface) and because electrons born in the cusp can only be scattered into the loss cone by collisions, the plasma resistivity within the cusp may support a significant potential difference axially along the cusp. Therefore, application of an external bias to the multipole structure may be sufficient to produce ion beam emission from the cusp. With an appropriate means of high density plasma production at the cusp, this biased nozzle effect can be used to gridlessly accelerate ions, providing a first step towards a potential electrostatic thruster. Because such a device lacks any kind of acceleration grid, it is more akin to a Hall thruster, rather than a gridded ion thruster.

\section{Microwave ECR Cusp Accelerator}

To realize a cusp accelerator device, microwave driven electron cyclotron resonance (ECR) was investigated as the plasma production mechanism within the cusp. ECR plasmas have many distinct advantages over traditional DC, hollow cathodebased sources ${ }^{3,4}$. These include electrodeless plasma production and instant on operation. Unlike hollow cathode discharges, ECR plasmas can operate with a variety of different propellants, including certain chemically reactive species. Furthermore, environmental protocols associated with hollow cathodes, which typically increase cost and handling complexity, are completely eliminated. Furthermore, in the context of a hall thruster, ECR could provide the means to separate plasma production (absorbed microwave power) from ion acceleration (applied device bias). This could potentially lead to a substantial increase in thruster efficiency.

Microwave-driven multipole sources commonly have difficulty producing very dense plasma over magnetic cusps. This usually arises because the microwave power is launched from an external source and must invariably approach resonance from the low field side. As a result, microwaves typically encounter the $\mathrm{R}$ wave cutoff before resonance and would otherwise be reflected if it were not for tunneling into the upper hybrid resonance. Even in the case of tunneling, absorption at the upper hybrid resonance can only occur if the local plasma frequency is less than the microwave frequency. Otherwise, the microwave power will simply be reflected. These reflection and resonance processes are illustrated in the Clemmow-Mullaly-Allis (CMA) diagram shown in fig. 2. The arrow represents the path taken by an externally launched microwave approaching ECR from the low field side. Note that in fig. 2, increasing plasma frequency, $\mathrm{w}_{\mathrm{pe}}$, and increasing electron cyclotron frequency, $\mathrm{w}_{\mathrm{ce}}$, represent increasing plasma density and magnetic field respectively.

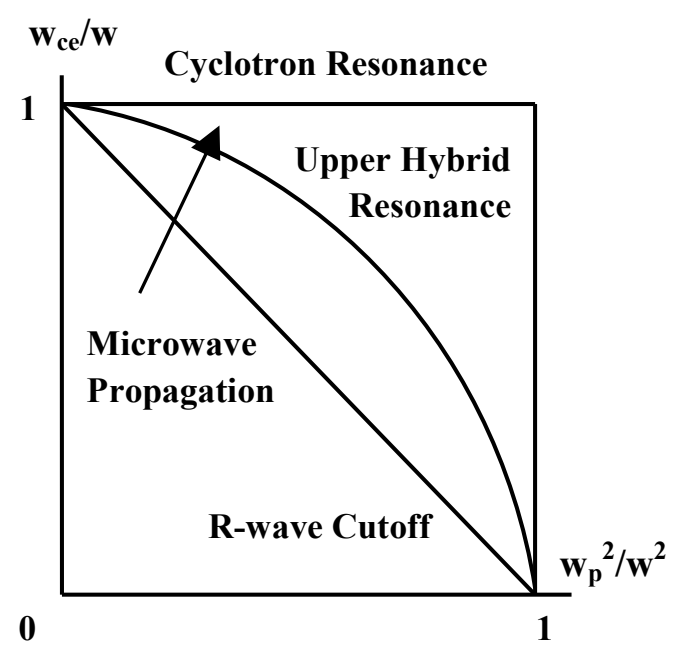

Figure 2. CMA diagram showing the $R$ wave resonance and cutoff. Microwaves traveling from low to high magnetic field will hit the $R$ wave cutoff before resonance.

Efficient ECR production can be achieved, however, if an exciting antenna is placed at the root of the cusp. At this location, the field strength is larger than that required for resonance, allowing high to low field microwave propagation. This approach is employed in so-called distributed ECR plasma sources $^{5}$, which have demonstrated over-dense plasma production deep inside magnetic cusps. Here overdense denotes the condition where the local plasma frequency exceeds the microwave frequency.

In this work, we employ the method of distributed ECR to produce a bias driven ion source. This source is formed from a combination of the natural nozzle effect shown in fig. 1a and an externally applied voltage. By applying a bias to the entire device, the plasma potential of the cusp region can be lifted up, allowing ions to accelerate out of the cusp to lower potential surfaces. Furthermore, it is expected that the ion Larmor radius for field strengths of interest is larger than the characteristic scale length of inhomogeneity, particularly at those regions downstream of the cusp. Therefore, ions should be largely unmagnetized and easily expelled by the resulting DC electric field. 
The objectives of this work are twofold: first, to demonstrate ECR plasma production at the magnetic cusps, and second, to demonstrate control over the energy of ions emitted from these cusps. To this end, we present measurements of extractable ion and electron current as well as ion energy distributions as a function of device bias voltage. For the ion energy measurements, beam width and bias voltage acceleration efficiency are also documented.

\section{Source Design and Experimental Setup}

\section{A. Microwave Device Design}

The primary design goal of the microwave device was to apply the concept of distributed ECR to create a bias driven ion source. A simplified diagram outlining this mechanism is shown below in fig. 3. As in typical DECR devices, an antenna was used to excite ECR over a permanent multipole magnet configuration. In this configuration, the multipole structure was housed in a thin rectangular cavity that was isolated from ground. Argon gas was fed directly into the cavity from an isolated feed system that could provide up to $32 \mathrm{sccm}$. The outside chamber pressure was measured using a thermocouple gauge for pressures down to $1 \mathrm{mTorr}$ and an ion gauge for pressures down to $10^{-5}$ Torr.

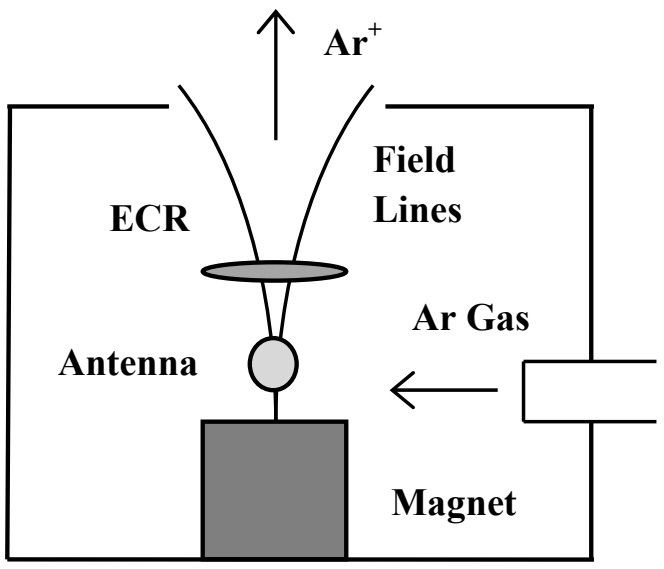

Figure 3. Microwave ECR device setup. The cusp geometry provides a natural nozzle effect to expel ions produced in the ECR production zone.

Electron and ion current emitted from the cusp plasma was extracted through slots of various sizes and located at various positions relative to the center of the magnetic cusps. The variety of slots and their placement depended on the particular experiment. The detailed results of these slot extraction studies are presented in the results section.

The microwave frequency used in this work was $2.45 \mathrm{GHz}$, which was resonant with electron cyclotron motion at a magnetic field strength of 875 G. Microwave power was provided by two copper antennas that were fed into the device from a 2.45 $\mathrm{GHz}$ microwave generator and positioned above permanent Samarium-Cobalt ( $\mathrm{SmCo}$ ) magnets such that the $875 \mathrm{G}$ resonance line was located vertically downstream of the antennas. The exact alignment of the antennas over the magnetic columns is shown in fig. 4. The device contained 5 columns of magnets, with 3 magnets per column. The magnet columns were all aligned with the same polarity so that an additional cusp structure was formed in the space between them. This so called virtual cusp exhibits mirror force effects similar to those of a true cusp, and could provide an alternative mechanism for ion extraction. Therefore, part of the experimental goal was to investigate beam extraction from the virtual cusp and compare it with extraction over the true cusp. To achieve this goal, the copper antennas were aligned perpendicular to the columns to allow possible excitation at both true and virtual cusp resonance lines. The magnet orientation used in this work is in contrast to conventional cusp configurations, like that shown in figure $1 \mathrm{~b}$, in which the magnets are aligned with alternating polarity.

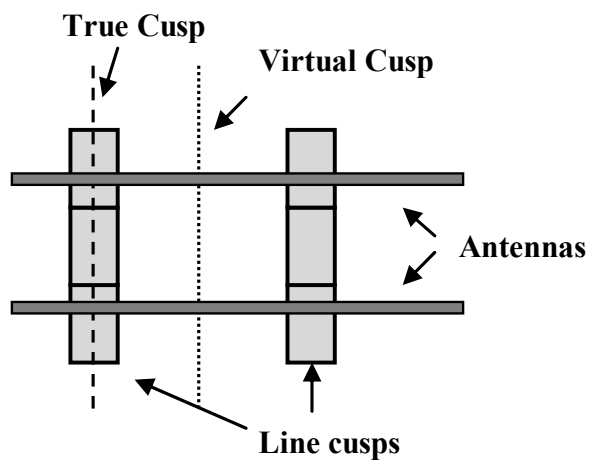

Figure 4. Magnet and antenna orientation inside the ECR device. The locations of the true and virtual cusps are shown in dashed lines.

Because the dimensions of the apparatus were smaller than the wavelength at $2.45 \mathrm{GHz}(12.2 \mathrm{~cm})$, the electric field was treated quasistatically as an oscillating electric field rather than that of a traveling wave. In this respect, concerns regarding wave propagation to the ECR zone were completely mitigated. The antenna merely needed to be placed such that the field oscillations at ECR were sufficiently high to break the gas down, energize the electrons, and sustain the plasma. In many respects this required that the antenna be placed in a region of high field strength where the oscillating electric field could interact with the electrons in the heating zone 
while at the same time assure that plasma production did not occur immediately at the antenna, which could have given rise to high density plasma exposure at the antenna and eventually led to erosion. The separation between antenna and intense plasma production zone also avoided plasma shielding that would tend to localize the volume of plasma production to a thin layer around the antenna.

\section{B. Current Extraction Measurements}

A test was performed to determine the optimal location for current extraction, comparing the performance between true and virtual cusps. Compared to the true cusp, the geometry of the virtual cusp has a smaller electron loss width and a lower field gradient in the vicinity of the cusp. If electrons created over the true cusp were to flow along field lines into the virtual cusp region, the lower magnetic field gradient might make electron collection at the anode surface below the virtual cusp favorable. Since extractable ion current is usually limited, through charge neutrality, by electron collection to the device wall, higher electron collection might lead to higher ion emission. One drawback of this effect is that the reduced impedance to electron flow will result in a lower sustainable potential drop axially along the cusp, resulting in reduced ion acceleration. In addition, the increased loss of hot electrons could lead to a decrease in ionization efficiency. Overall, comparing the performance of the two cusps provides a first insight into the effect of electron loss width and magnetic field gradient on ion beam emission from the cusp.

To individually assess the extractable current over each type of cusp, separate tests were performed with a single slot cut over either the true or virtual cusp. The baseline size of the slots was chosen to be on the order of the width of the SmCo magnets $(\sim 0.5 \mathrm{~cm})$, so that only the plasma from a single magnetic cusp would be sampled. The slot size over the real cusp was $5 \mathrm{~cm}^{2}$, while that over the virtual cusp was 3.5 $\mathrm{cm}^{2}$. A collector plate, $5 \mathrm{~cm}$ by $10 \mathrm{~cm}$, was positioned $2.5 \mathrm{~cm}$ above the exposed slot of the device. The total distance between the collector and the surface of the magnets was $4 \mathrm{~cm}$. The entire discharge chamber cavity was then biased relative to the collector. Either electron or ion current could be extracted depending on the bias applied to the discharge device. The current incident on the collector plate was measured as a function of the applied bias. I-V traces of both electron and ion current were taken at various levels of absorbed microwave power, background vacuum chamber pressure, and argon mass flow rate.

\section{Ion Energy Measurements}

Ion current extraction measurements provide insight into the available plasma current within the device but do not provide details of the extracted beam's energy distribution. In this regard, ion energy measurements are necessary to determine how the emitted ion beam energy correlates with the applied bias voltage to the device. The objective in this case is to optimize the discharge so that most of the applied bias voltage appears along the cusp region where it can accelerate the ions rather than in a localized region across the collector sheath. In this work, ion energy measurements were taken over a wide range of background chamber pressure, device bias voltage, and absorbed microwave power. Other observations relevant to beam quality were made, which include beam efficiency, defined here as the ratio of the observed beam peak voltage to the applied bias voltage, and normalized beam width, which is defined as the ratio of the full width half maximum of the beam to the observed beam peak voltage.

The ion beam energy distribution was measured using a gridded retarding potential analyzer (RPA). A detailed description of the theory of probe operation can be found elsewhere ${ }^{6}$, but the main principle of operation is that by biasing the probe to a voltage $\varphi$, one can allow only ions with energy greater than $e \varphi$ to pass through the probe to a current collector. The probe produces an IV curve, which can be differentiated to yield the energy distribution $\mathrm{f}(\mathrm{e} \varphi)$ of the incident beam in terms of the ion mass $M$, the electronic charge $\mathrm{e}$, the transmission coefficient of the probe grid $\tau$, and the area of the probe collector A,

$$
f(\mathrm{e} \varphi)=\frac{\mathrm{M}}{\mathrm{A \tau e}^{2}} \frac{\mathrm{d} I c}{\mathrm{~d} \varphi}
$$

In this experiment, there was no cathode to neutralize the emitted ion beam; instead, the beam was neutralized at a grounded collector plate. The RPA was placed immediately downstream of a hole cut in the collector plate so that the front of the probe lay in the same plane with the collector plate. The entrance grid of the RPA was placed $2.5 \mathrm{~cm}$ above a small 3 $\mathrm{mm}$ orifice hole cut in the top of the discharge chamber body. The purpose of this small hole size was to limit current emitted by the source plasma. This reduced the current flow into the RPA in order to keep the Debye length large compared with grid spacing within the RPA, thereby allowing the probe to operate essentially in the single particle limit.

The setup of the probe used in this work, shown in fig. 5, consists of three grids and a collector, each 
biased separately. The front grid was shorted to the body of the RPA and grounded so that it remained at the same potential as the collector plate. The middle grid was varied to restrict ion flow to the collector, which was set at $-20 \mathrm{~V}$ to attract all ions with sufficient energy to pass through the middle grid. The third grid was set at $-20 \mathrm{~V}$ relative to the collector to prevent the escape of secondary electrons that are created by ion bombardment on the collector. Without the third grid, these electrons could escape the probe and be counted as erroneous ion current to the collector.

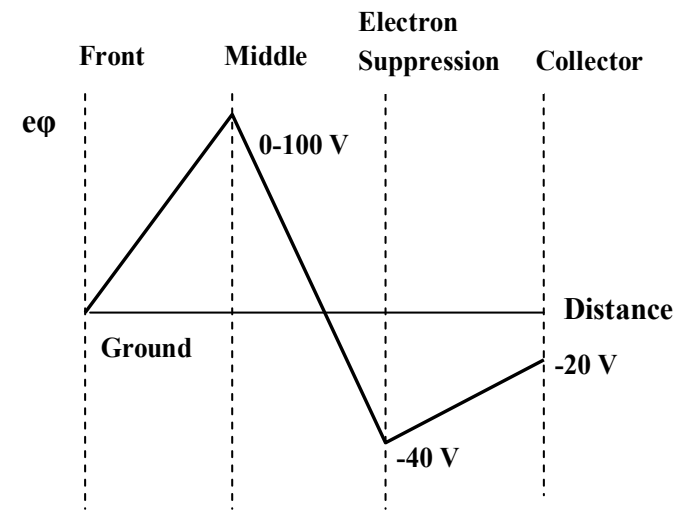

Figure 5. Grid voltage settings used in RPA measurements. Ions enter the front grounded grid (left) and travel toward the collector.

\section{Results and Analysis}

\section{A. General Operation}

The microwave ECR device was observed to operate successfully using both single and double antenna excitation. In each case, the plasma turned on without the aid of a seed plasma or high voltage breakdown and could operate down to a minimum of 10 watts per antenna. Once breakdown occurred, the reflected microwave power could be easily reduced to less than $10 \%$ of the total power by a 3-stub tuner and remained well matched regardless of small changes made to power and pressure. The reported absorbed input power represents an upper limit since it has been observed that some fraction of the input power is actually transmitted out of the device, presumably via the exposed current extraction area. In this respect, the exposed area may be acting as a radiating slot. This undesirable coupling is believed to be minimal. Therefore, all references to absorbed power will simply refer to the total power minus the reflected power. It should be further pointed out that the leakage power may actually be removable by the re-orientation of the slot. Slot re-orientation along with magnetic circuit optimization is left to future work.

The cusp plasma was observed to be stable over the pressure range $0.5 \mathrm{mT}$ Torr to $100 \mathrm{mTorr}$. At high pressures, the plasma was heavily localized around the antenna but at low pressures ( $\sim 1$ mTorr), the plasma was observed to spread out over a much larger volume and couple to multiple line cusps simultaneously. This observation highlights two desirable attributes: 1) the source favors low pressure operation, which translates into low required gas flow-rates and 2) the device could easily be scaled up or down by adding or removing magnetic cusps.

\section{B. Current Extraction}

\section{General Current Extraction Results}

Current extraction measurements were performed with both antennas running simultaneously over the pressure range $1.5 \mathrm{mT}$ Torr to $20 \mathrm{mTorr}$. The extraction tests were performed by removing a portion of the discharge chamber to allow for the placement of slotted apertures. As discussed in the setup section, the apertures exposed either a single true cusp or a single virtual cusp. Figure 6 shows I-V curves taken over the true cusp slot in the ion current regime for two typical operating power levels, $40 \mathrm{~W}$ and $70 \mathrm{~W}$. In general, the ion current was observed to increase monotonically with decreasing pressure. Operation at chamber pressures below 1.5 mTorr was intentionally avoided to prevent excessive heat loading on the antenna. Future antenna designs will employ stronger metals to prevent melting and sagging. In addition, aluminum oxide covers will be applied to prevent excessive antenna erosion.

The substantial increase in current observed in fig. 6 at low pressure is readily explainable by considering collisions within the ECR production zone. Ordinarily, one would expect a monotonic increase in plasma density as the pressure is reduced. This should occur up to the point where the product of the ionization reaction rate coefficient $(<\sigma v>)$ and the neutral density, $\mathrm{n}_{\mathrm{o}}$, reaches a maximum. Thus, even though the neutral density is decreasing, the plasma density can still be increased if the ionization product is sufficiently large. As the pressure is reduced, the electron will be able to execute more Larmor orbits, gaining more energy between collisions. This increase in absorbed energy can result in the required increase in $\langle\sigma v\rangle$ to yield an overall increase in plasma density.

Furthermore, one can consider the effect of changing pressure on the hall parameter, $\mathrm{w}_{\mathrm{ce}} / \mathrm{v}_{\mathrm{e}}$, 
which is the ratio of the electron cyclotron frequency to the electron collision frequency.

(a)

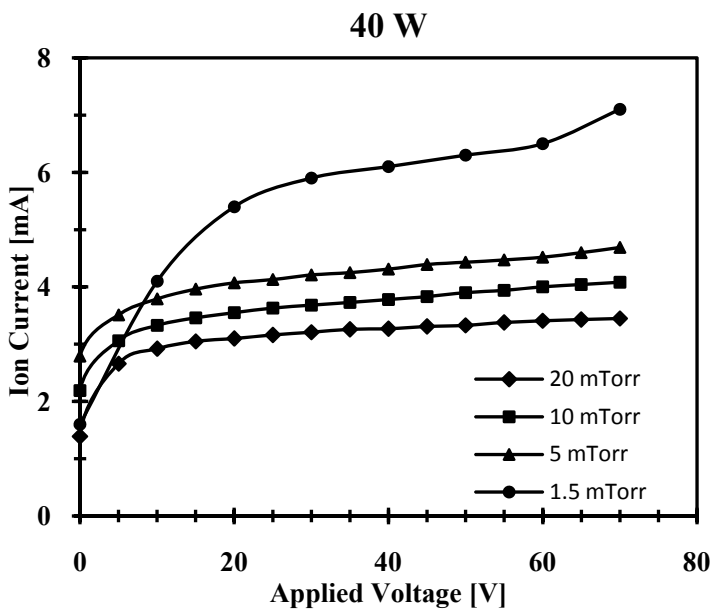

$70 \mathrm{~W}$

(b)

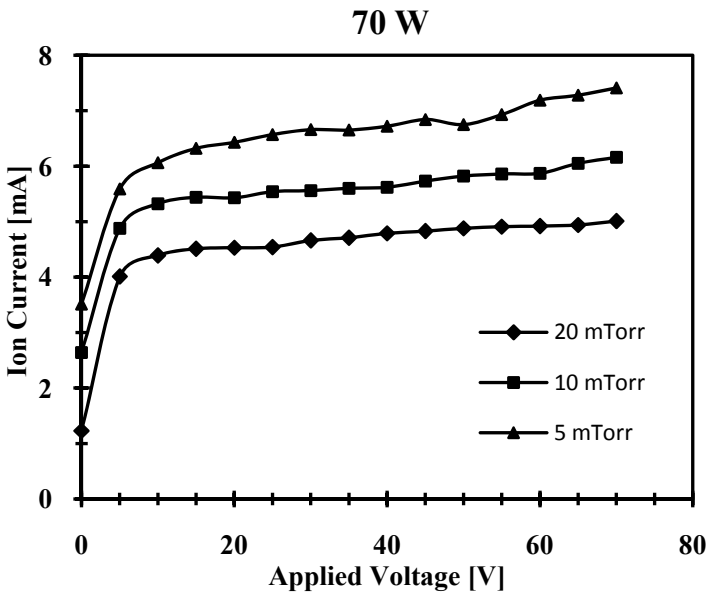

Figure 6. Ion extraction over the true cusp a.) $40 \mathrm{~W}$ absorbed power. b.) $70 \mathrm{~W}$ absorbed power.

In changing from 5 mTorr to 1.5 mTorr, the hall parameter in the ECR production zones decreases by a factor of approximately 3 . This means that between consecutive collisions, the microwave field can do approximately 3 times more work on the orbiting electron. Since density scales with absorbed power, one would also expect the ion current to increase by a factor of 3. However, the actual observed increase in ion saturation current is only 1.5 , half that expected. This is likely due to the fact that the ultimate extractable ion current is limited by the electron current collected at the discharge chamber wall. That is, in order to preserve charge neutrality, the loss width for electron current does not permit such a large increase in available ion current.

From a qualitative standpoint, the transition to higher emitted current at lower pressures was observed to coincide with the formation of the aforementioned diffuse plasma over multiple cusps. This behavior is consistent with the phenomological model described above. As the pressure is reduced, electrons are accelerated between collisions to higher energies by both the microwave and the DC field. Electrons not falling in the loss cone are left to reflect between mirrors. Because these reflecting electrons have higher energies under low-pressure conditions, they are more likely to ionize and excite neutrals as they mirror between cusps, thereby giving rise to what appears to be a more uniform discharge.

Another interesting feature of the data in figure 6 is the collected ion current at $0 \mathrm{~V}$ applied bias. At every pressure condition, the collected ion current at $0 \mathrm{~V}$ is significant, in some cases even as high as $60 \%$ of the ion saturation current. This observation provides confirmation of the natural nozzle effect occurring within the cusp, which predicts that ions will tend to spill out of the cusp even without the aid of an external bias.

The data in fig. 6 also indicate that ion current saturates at relatively low voltages, around $20 \mathrm{~V}$. The maximum ion current measured over the two power levels was $8 \mathrm{~mA}$ at an extraction voltage of 70 V. Given that the exposed slot is $5 \mathrm{~cm}^{2}$, the lower limit of current density of plasma flowing out of the device is around $1.6 \mathrm{~mA} / \mathrm{cm}^{2}$. The actual emitted current density depends on the width of the ECR heating zone. The actual emission area is therefore expected to be considerably smaller than $5 \mathrm{~cm}^{2}$, which in turn would lead to a significantly larger emission current density. Modeling the size of this emission site is ongoing. Its dimensions depend not only on magnetic structure but also on microwave bandwidth. Measurements for $1.5 \mathrm{mTorr}$ at $70 \mathrm{~W}$ were not included because at such high power and low pressure, the copper antennas were observed to undergo significant thermal loading, which led to sagging and eventual grounding to the magnet surface. As a result, the low-pressure limit of operation was not fully explored at this power level. As mentioned earlier, antenna design modifications should alleviate this problem.

It should also be pointed out that current flow along the cusp axis is inherently space charge neutralized as it involves counter flowing ion and electron current. It is not until the beam emerges from the source that space charge effects are meaningful.

\section{Electron Current Measurements}

Extraction of electron current, which was obtained by biasing the source negative, is shown in fig. 7 for the same conditions as the true cusp. In contrast to ion extraction, electron current behavior 
did not change significantly over the measured pressure range and was observed to decrease slightly in magnitude with a decrease in pressure. Electron current reached up to $100 \mathrm{~mA}$ and $140 \mathrm{~mA}$ for the 40 $\mathrm{W}$ and $70 \mathrm{~W}$ conditions respectively, and was limited by the onset of significant arcing in the chamber at negative $70 \mathrm{~V}$ bias voltage, presumably initiated by the presence of the energetic electron beam. Overall, the emitted electron current was considerably higher than the emitted ion saturation current. The difference is due to the fact that the ions are not as magnetized as the electrons and are thus more easily collected at the device walls. This in turn allows the emission of electron current in order to satisfy charge neutrality. The trend of increasing electron current with increasing pressure is opposite to that observed in the ion extraction case.

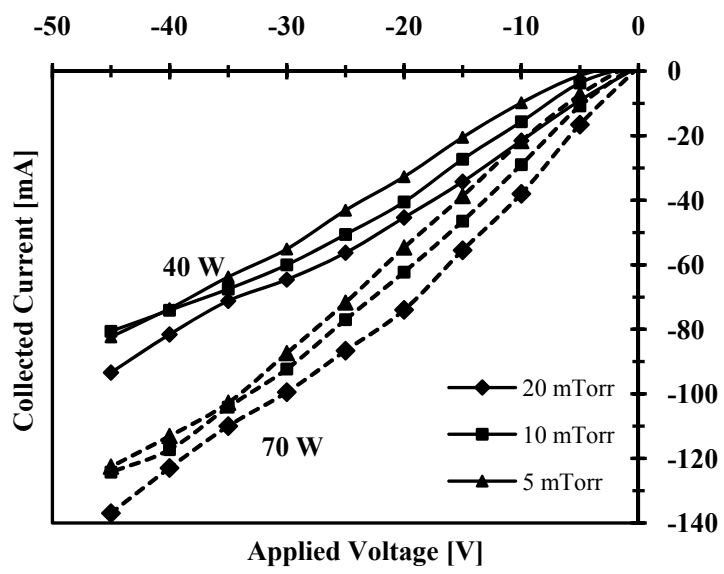

Figure 7. Electron extraction over the true cusp. Electron current was observed to be less sensitive to a change in pressure than ion current.

When extracting electrons, the increase in internal pressure presumably increases internally collected ion current. This is most likely due to the presence of impact ionization of electrons as they are driven out of the device by DC bias acceleration. Thus, in this case, the neutral density plays the largest role in determining the reaction rate. The fact that the current does not saturate over the investigated bias range, but rather increases linearly with voltage is further evidence in support of the presence of this impact ionization. In this respect, the electron current behavior with voltage and pressure is likely heavily influenced by the DC electric field.

\section{Virtual Cusp Measurements}

A current extraction test was performed with the exposed slot over the virtual cusp for comparison with real cusp measurements. Figure 8 shows I-V traces at $40 \mathrm{~W}$ absorbed power in the 1.5-20 mTorr range. It was previously mentioned that the slot area over the virtual cusp $\left(3.5 \mathrm{~cm}^{2}\right)$ was cut slightly smaller than that of the true cusp $\left(5 \mathrm{~cm}^{2}\right)$. To correct for this difference and provide a more realistic comparison between the two cusp measurements, the current extraction data in fig. 8 have been multiplied by the ratio of the true cusp slot area to that of the virtual cusp. This is motivated by the prediction that current density will be approximately uniform over slot areas on the order of the width of the magnet. After applying this compensation, the current collected over the real cusp is $30 \%$ larger at high voltages and $90 \%$ larger at low voltages. These observations indicate that the true cusp is better suited for ion extraction than the virtual cusp. This may be explained by the fact that the virtual cusp loss width is larger than that of the true cusp, leading to a corresponding higher loss rate to the device. Even though it was expected that a larger electron collection would allow the emission of more ions, it appears that the loss of hot electrons may have resulted in a lower ionization rate and thus a lower plasma density. This would account for the lower current density over the cusp.

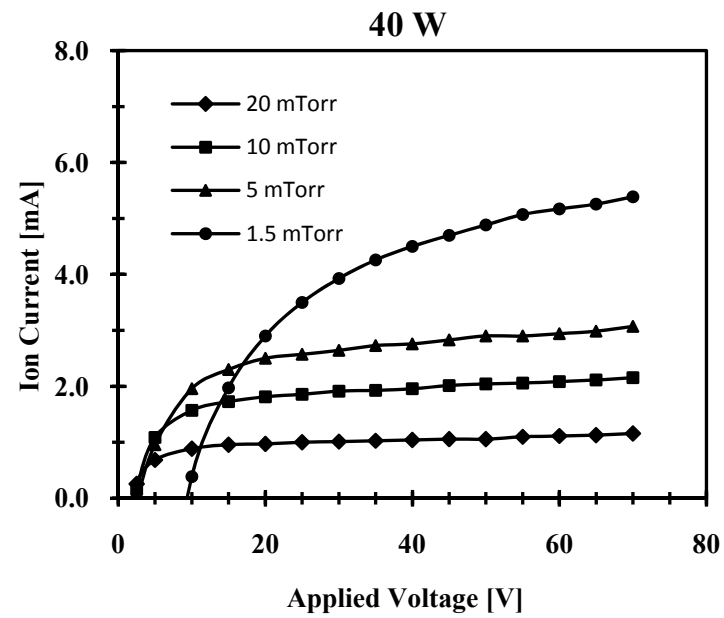

Figure 8. Ion extraction over the virtual cusp at $40 \mathrm{~W}$. Measured currents are corrected by the ratio of the true cusp area to virtual cusp area.

One point of similarity between the real and virtual cusp data is the increase in emitted ion current with decreasing pressure. The physical basis for this behavior was discussed previously. Just as in fig. 6, the extractable current is nearly doubled at $70 \mathrm{~V}$ when decreasing the pressure from 5 mTorr to 1.5 mTorr. One difference between the two cusp measurements is that in the case of the virtual cusp, the current extracted at $0 \mathrm{~V}$ bias voltage is negative where as the measured current over the true cusp was 
significantly positive at $0 \mathrm{~V}$. This indicates that ions may not be reaching high enough energies to leave the device, which is consistent with the previous explanation that virtual cusp electron losses may be too great to support a significant potential difference across the cusp. Overall, these current extraction results suggest that the microwave ECR device performs optimally at low pressures.

\section{Ion energy}

\section{General observation of Ion Beam Distributions}

Data was taken with the retarding potential analyzer (RPA) over the pressure range $1.5 \mathrm{mTorr}$ to $8 \mathrm{mTorr}$ at a two different power levels, $20 \mathrm{~W}$ and 40 W. Figure 9 shows RPA traces for various device bias voltages at $20 \mathrm{~W}$ absorbed microwave power and $1.5 \mathrm{mTorr}(2.5 \mathrm{sccm})$. At each value of bias voltage, the collected current begins to fall off sharply in the vicinity of the device bias voltage, indicating the presence of an ion beam. This was observed over a wide voltage range up to $90 \mathrm{~V}$.

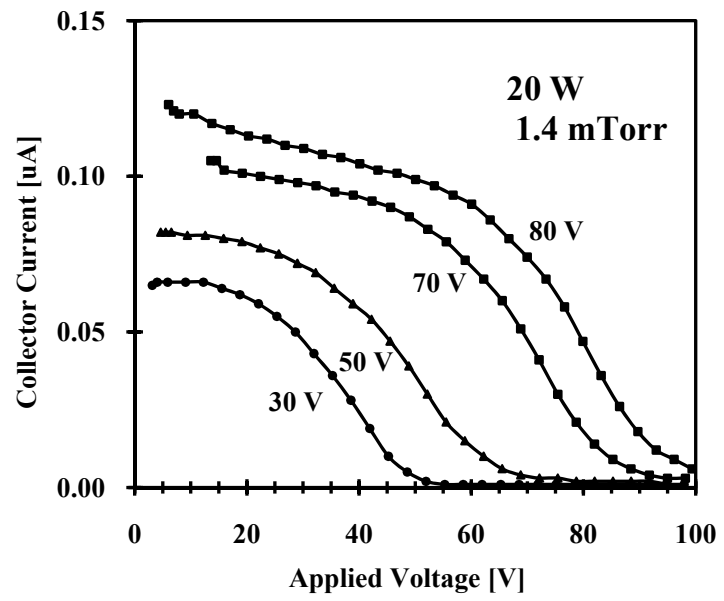

Figure 9. RPA traces. Traces taken at $20 \mathrm{~W}, 1.4$ mTorr, $2.5 \mathrm{sccm}$.

A peculiar effect was observed, however, at $20 \mathrm{~V}$ bias voltage. The RPA trace at this condition indicated the presence of energetic ions far exceeding the bias voltage. The presence of this structure in the RPA trace is still not well understood though it may be related to a mode change at the antenna driven heating site or possibly even charge exchange occurring within the device. It is not clear why it happens only at the $20 \mathrm{~V}$ case, but if the plasma potential is very high under these conditions, then ions will fall out at potentials higher than the bias voltage. A rigorous test of this explanation will be included in future experiments using emissive probes to map the local potential over the cusp.
The ion beam energy distributions for both power levels were calculated using eq. 1 and normalized to unity; a few representative peaks from each power level are displayed in fig. 10. These curves confirm that significant beams are present in the voltage range 30-80 V.
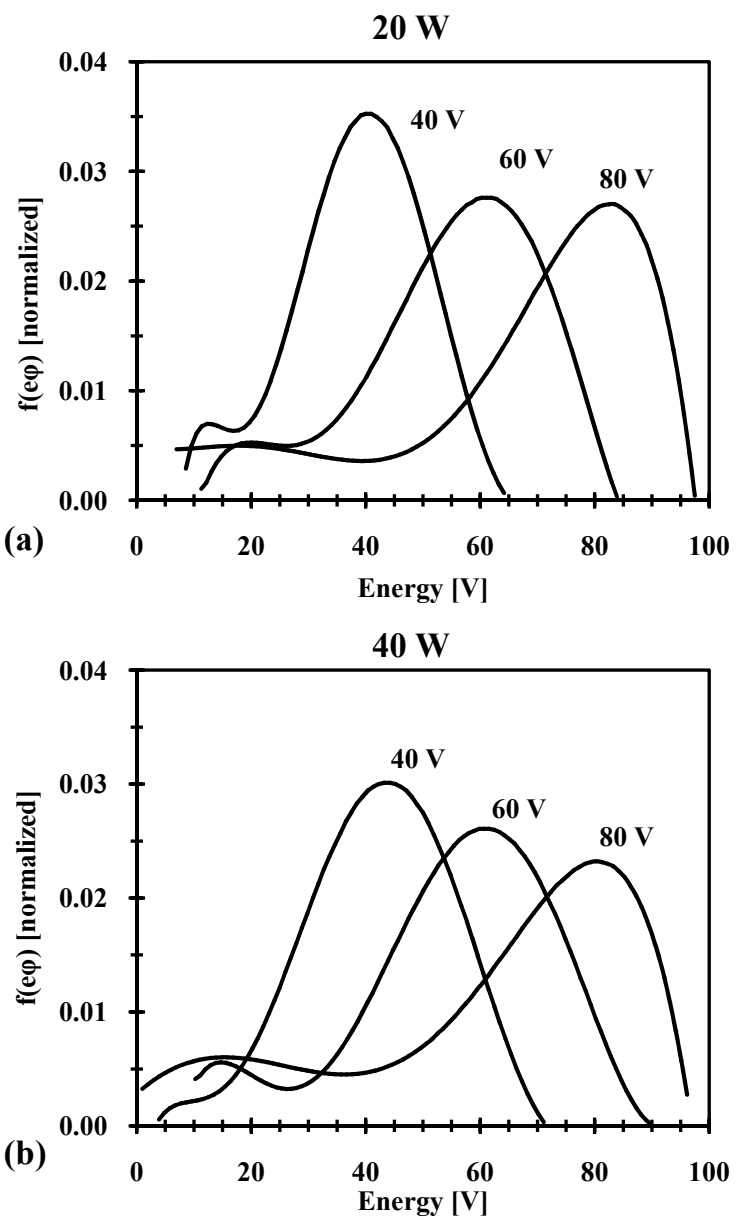

Figure 10. Ion energy distributions. Traces taken at $1.4 \mathrm{mTorr}, 2.5 \mathrm{sccm}$. a.) $20 \mathrm{~W}$ b.) $40 \mathrm{~W}$,

Moreover, these beams have peaks that correspond very closely to the applied device discharge voltage, in most cases to within a few volts. If the acceleration efficiency is defined as the ratio of the beam peak voltage to the applied bias voltage, then for all observed beams, the efficiency is very near $100 \%$. This suggests that a controllable potential difference is being created over the cusp and that ions produced in this region are being efficiently accelerated out of the cusp plasma. The distributions in fig. 10 also display a small population of ions at low energy $(\sim 20$ $\mathrm{V})$. These low energy ions could be the result of collisional processes such as charge exchange 
occurring within the device. These effects are discussed in a later section.

\section{Beam width measurements}

The ions in the observed beams are expected to be created primarily over a finite length along the cusp axis, centered in the vicinity of the heating zone. As a result, the observed ion beams should have considerable width or spread in energy. An additional source of broadening could be electron impact ionization within the cusp sheath. The resulting beam width can be measured directly using the distributions in fig. 10. For this analysis, normalized beam width was defined as the full width half maximum (FWHM) of the observed beam peak divided by the beam peak voltage. Figure 11 shows beam width plotted as a function of beam peak voltage for the cases taken at $20 \mathrm{~W}$ and $40 \mathrm{~W}$.

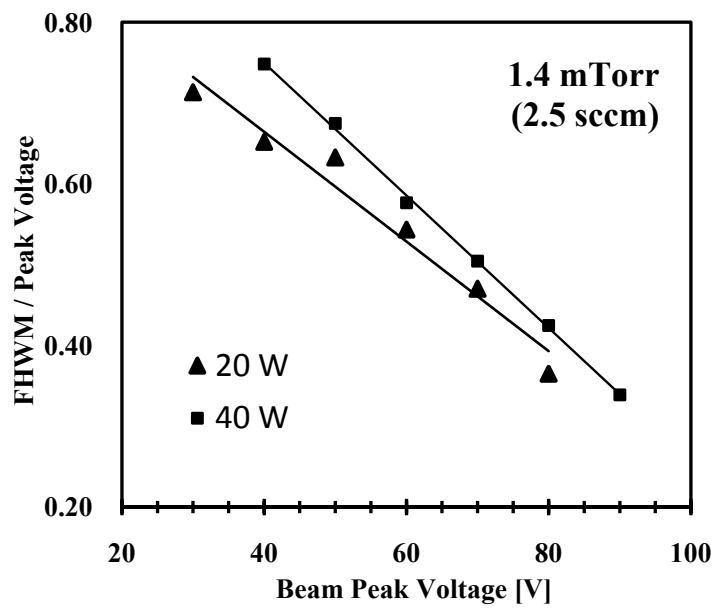

Figure 11. Ion beam width over the voltage range 30-80 V. FWHM is normalized to beam peak voltage.

The observed trend is linear, with beam width decreasing as bias voltage is increased. This result is consistent with common observations that narrow width beams are difficult to form at low discharge voltages because space charge effects tend to cause dispersion $^{7}$. Moreover, the similarity between the 20 $\mathrm{W}$ and $40 \mathrm{~W}$ cases suggests that the width is not broadening appreciably with an increase in power. In addition, the FWHM itself was observed to stay fairly constant over the range of applied voltages. In the 20 $\mathrm{W}$ case, the FHWM changed by only $16 \%$ when increasing the voltage from $30 \mathrm{~V}$ to $80 \mathrm{~V}$. Overall, the observation of decreasing beam energy spread with little increase in FWHM at higher discharge voltages is promising for future evolutions of the device, which will be expected to run at discharge voltages closer to that of a hall thruster (300-500 V). Simple extrapolation to higher voltages suggests that beams of comparable spread in energy may be expected in this range.

\section{Effects of pressure}

A more general characterization of ion beams at low power $(20 \mathrm{~W})$ was performed to assess the sensitivity of the beam to background chamber pressure. This was performed over the range 1.5 mTorr to 8 mTorr. Beam distributions are shown below in fig. 12 for two discharge voltages, $50 \mathrm{~V}$ and $70 \mathrm{~V}$, taken at 20 $\mathrm{W}$ power.

$70 \mathrm{~V}$
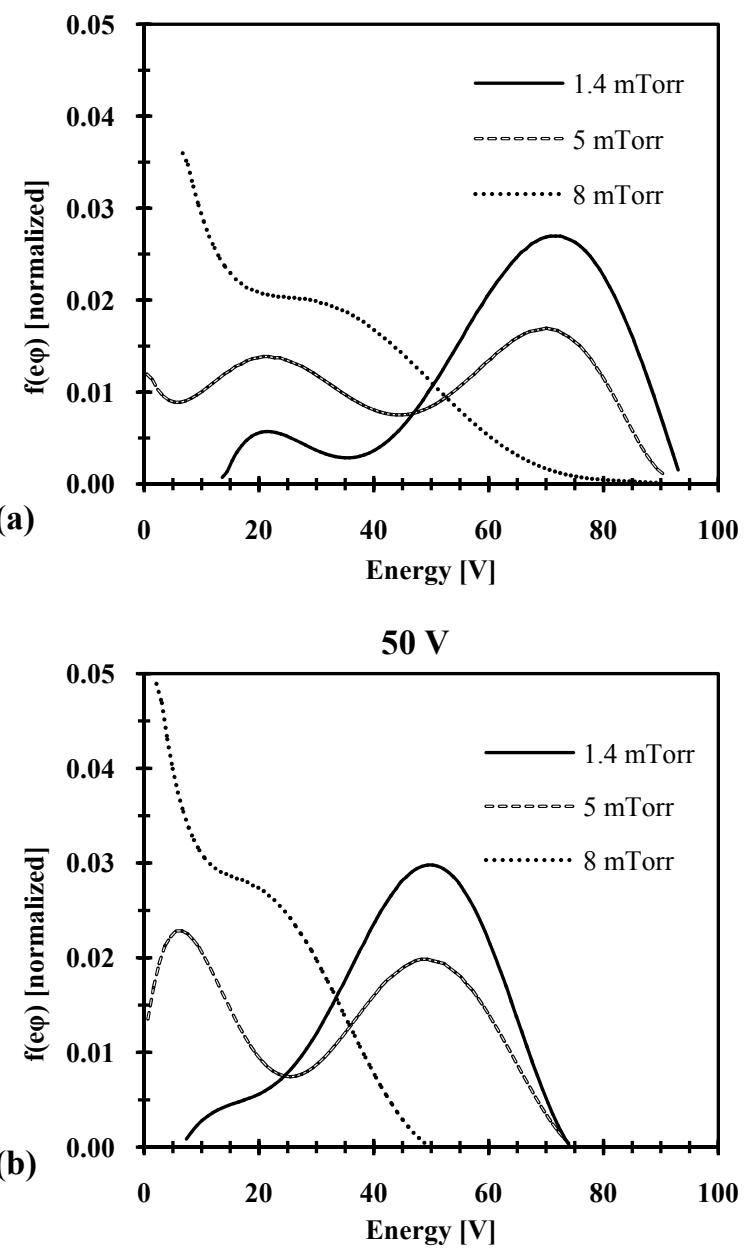

Figure 12. Ion energy distributions over the pressure range $\mathbf{1 . 5 - 8}$ mTorr. a.) $70 \mathrm{~V}$ bias voltage. b.) $50 \mathrm{~V}$ bias voltage. The increase in pressure leads to significant beam degradation.

These traces illustrate the dispersive effect of increasing the background pressure from $1.5 \mathrm{mTorr}$ to $8 \mathrm{mTorr}$. Though a beam is still observed at 5 
mTorr, a significant majority of the collected beam current now appears centered at a secondary low energy peak. When the pressure is raised to $8 \mathrm{mTorr}$, the well defined ion beam structure (energy profile) completely disappears and is replaced by a broad high energy tail that extends out to the bias voltage. The degradation of the ion beam at high pressures is most likely due to the presence of either momentum transfer collisions or resonant charge exchange collisions. These may occur either inside the chamber or directly outside of the orifice in the space between the device and the RPA.

Cross sections for momentum transfer and resonant charge exchange cross sections are readily available for argon in the relevant energy range 10-50 $\mathrm{eV}$. Raju ${ }^{8}$ and Rapp ${ }^{9}$ give cross sections for momentum transfer (MT) and charge exchange (CEX) typically on the order of $10^{-20} \mathrm{~m}^{2}$ and $10^{-19} \mathrm{~m}^{2}$ respectively, indicating that over the specified energy range, charge exchange will be the dominant collisional process. Assuming $300 \mathrm{~K}$ for the ion temperature, the mean free paths for MT and CEX at $1 \mathrm{mTorr}$ are around $20 \mathrm{~cm}$ and $7.5 \mathrm{~cm}$ respectively, which are both much larger than the separation between the source and the RPA as well as the characteristic length scale within the source $(\sim 2-4$ $\mathrm{cm})$. As the pressure is raised to $5 \mathrm{mTorr}$, however, the CEX mean free path drops down to about $1.5 \mathrm{~cm}$ while the MT mean free path is still large at about 5-9 $\mathrm{cm}$. This is consistent with the observation of a second ion population at 5 mTorr. Ions travel through the sheath a distance roughly equal to the CEX mean free path before undergoing a CEX reaction. As a result, the second ion population should be centered at the average energy that is gained by the newly formed low energy ions traveling through the remainder of the sheath. This explanation is also consistent with the observation that these secondary peaks change with discharge voltage. Due to a weak dependence on energy, the CEX mean free path can be treated as approximately constant over the relevant range of discharge voltage. However, a change in the discharge voltage also changes the sheath voltage drop across the CEX mean free path, thus changing the average energy of the secondary population and causing the observed shift. When the pressure is increased to $8 \mathrm{mTorr}$, both CEX and MT mean free paths are substantially lower than the ion transit length, so one would expect very large spread in the beam. In fact, this is confirmed by fig. 12; the beam has completely transitioned to a high energy tail in both the $70 \mathrm{~V}$ and $50 \mathrm{~V}$ case.

The above calculations assume that the internal pressure within the device is equal to the chamber pressure. In reality, the constant flow of neutral gas into the device will give rise to a finite conductance,
$\mathrm{C}$, across the $3 \mathrm{~mm}$ diameter ion current extraction orifice. This will result in a finite pressure difference between the internal device pressure, $\mathrm{P}_{\text {in }}$, and the outer vacuum chamber pressure, $\mathrm{P}_{\text {out }}$. This pressure difference will determined by the hole conductance, $\mathrm{C}$, and the total throughput, $\mathrm{Q}$, associated with the argon gas flow by the standard pumping equation ${ }^{10}$,

$$
P_{\text {in }}-P_{\text {out }}=\frac{Q}{C}
$$

The above relation will give rise to higher pressure inside the device, leading to a subsequent increase in all mean free paths, and thus higher beam degradation. Taking all of these calculations into account, the following observation can be made: if one assumes that the acceleration takes place completely in the cusp region, then the majority of scattering collisions, and thus energy dispersion, takes place inside the source. This makes sense when considering the increase in mean free paths within the device. In the preceding discussion, it was observed that the secondary ion peak moves as a function of discharge voltage. This effect is explicitly shown in fig. 13, which shows a plot of ion energy distributions at 5 mTorr for different discharge voltages. From this figure, it is clear that both ion peaks move as a function of bias voltage.

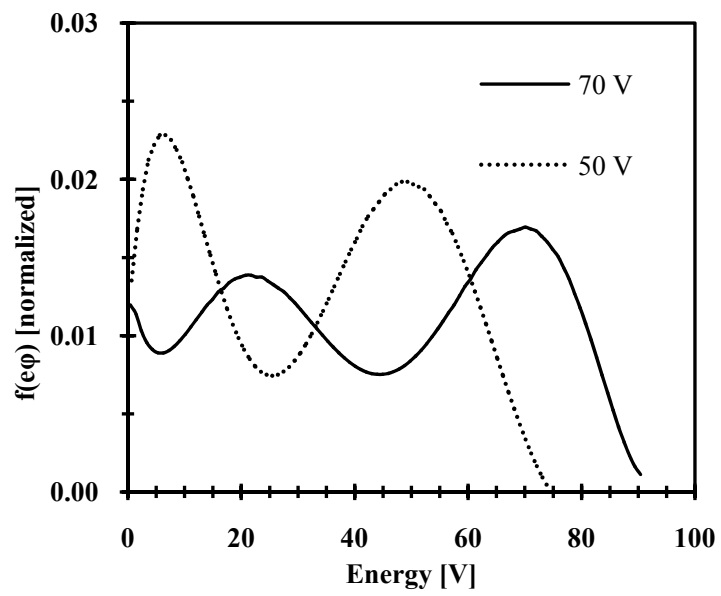

Figure 13. Ion energy distributions at $5 \mathrm{mTorr}$, $20 \mathrm{~W}$ power. Both peaks change as a function of discharge voltage.

\section{Conclusions and Future Work}

In this effort, we have demonstrated the ability to create controllable potential differences in an ECR cusp plasma and induce ion beam emission via the combined effect of an external bias and cusp mirror 
forces. Energy measurements taken with an RPA indicate good conversion from imposed bias voltage to actual peak energy. However, it is still unclear over what length of the cusp this potential difference appears. Detailed measurements of plasma potential will need to be performed directly within the cusp at varying distances above the magnet and correlated with observed beam energy.

The ion current and beam energy measurements presented in this paper were all performed with the same cusp geometry and antenna orientation. Given that the microwave coupling of the ECR plasma is so sensitive to the location of the antenna in relation to the magnetic field, further experiments will have to be performed to optimize current emission as a function of antenna position. In addition, the number magnets and their orientation will be varied to produce optimum plasma production above the cusp.

The cusp plasma approach discussed in this paper can be used as a means to produce and accelerate ions for thruster applications. Long term future work will involve replacing the current collector plate with a cathode in order to neutralize the emitted ion beam. This electron source will also help force electrons into the cusp region to boost the maximum potential difference that can be supported over the length of the cusp. With this addition, the thruster could evolve into something similar to a hybrid thruster: with the gridless acceleration and charge neutralized beam extraction of a hall thruster, but with the separation of plasma production and ion acceleration akin to a gridded ion thruster. Overall, the data presented in this paper indicates that this approach is scalable, making it applicable to micro-propulsion applications as well as higher power options.

\section{References}

${ }^{1}$ Wolf, B., Handbook of Ion Sources, CRC Press, Boca Raton, 1995.

${ }^{2}$ Limpaecher, R., MacKenzie, K. R., "Magnetic Multipole Containment of Large Uniform Collisionless Quiescent Plasmas," Rev. Sci. Instruments, Vol. 44, No. 6, June 1973, pp. 726-731.

${ }^{3}$ Kim, S., Itoh, Y., Satori, S., "Endurance Test of Microwave Engine," AIAA 2004-4126, 11-14, July. 2004.

${ }^{4}$ Takao, Y., Miyamoto, T., Yamawaki, K., "Development of ECR Microwave Discharge Ion Thruster," Vacuum, Vol. 65, 21 May. 1984, pp. 361-366.

${ }^{5}$ Pichot, M., Durandet, A., "Microwave Multipolar Plasmas Excited by Distributed Electron Cyclotron Resonance: Concept and Performance," Rev. Sci. Instruments, Vol. 59, No. 7, July. 1984, pp. 1072-1075.

${ }^{6}$ Hutchinson, I. H., Principles of Plasma Diagnostics, Cambridge Univ. Press, New York, 1987.

${ }^{7}$ Humphries, S., Principles of Charged Particle Acceleration, John Wiley \& Sons, New York, 1999.
${ }^{8}$ Raju, G.G., Gaseous Electronics, CRC Press, Boca Raton, 2006.

${ }^{9}$ Rapp, D., Francis, W.E., "Charge Exchange between Gaseous Ions and Atoms," Journal of Chemical Physics, Vol. 37, No. 11, Dec. 1962, pp. 2631-2645.

${ }^{10}$ O'Hanlan, J.F., User's Guide to Vacuum Technology, John Wiley \& Sons, New York, 1989. 